\title{
Polarfuchs (Kolumne)
}

\section{Luisa von Albedyll}

Alfred-Wegener-Institut, Helmholtz-Zentrum für Polar- und Meeresforschung, Bremerhaven, Deutschland

Correspondence: Luisa von Albedyll (luisa.von.albedyll@awi.de)

Published: 21 May 2021

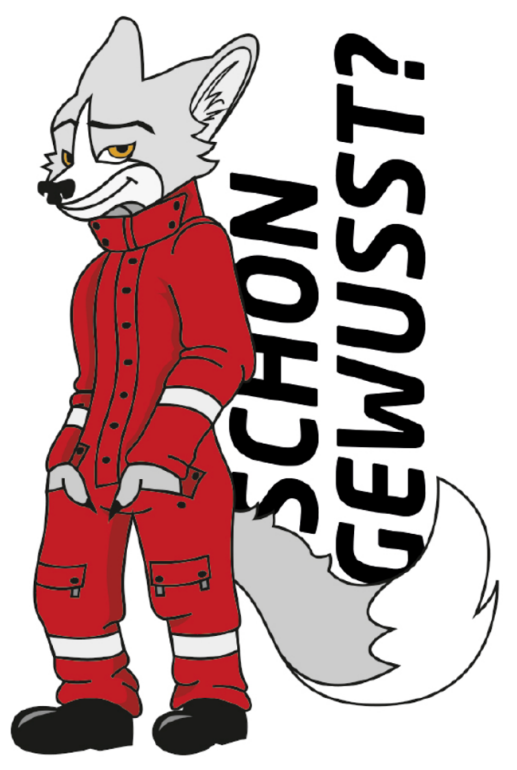

\section{Auf dünnem Eis - Der Polarfuchs am Nordpol}

Im Oktober 2020 haben viele Polarforschende mit Sorgenfalten im Gesicht das Meereis in der Arktis beobachtet - der Arktische Ozean fror vor allem in den sibirischen Randmeeren nicht wie gewohnt zu dieser Jahreszeit zu. Die Ausdehnung des Meereises war die niedrigste seit 41 Jahren, die je im Oktober gemessen wurde.

Dass die Ausdehnung des Eises immer geringer wird, wissen wir schon länger. Aber wussten Sie, dass das verbliebene Eis auch immer dünner wird?

\section{Ist die Abnahme der mittleren Eisdicke ein Problem?}

Ja, denn im Sommer schmilzt das dünne Eis viel schneller und früher. Der eisfreie Ozean nimmt dann über einen län- geren Zeitraum im Sommer die Energie der Sonne auf und gibt sie im Herbst und Winter wieder an die Atmosphäre ab. Das erwärmt die Luft und schwächt u.A. den Temperaturunterschied zwischen den polaren und den mittleren Breiten ab. Viele Forscher vermuten, dass das unser Wetter in den USA und in Europa verändert. Wenn der Temperaturunterschied schwächer wird, bewegt sich das Band aus Winden, das die warmen und kalten Luftmassen trennt, langsamer. Dadurch werden die Schleifen, in denen es sich bewegt, nach Norden und Süden größer. Das lässt den warmen und kalten Luftmassen mehr Zeit in die mittleren Breiten zu strömen und begünstigt Extremwetterereignisse bei uns, wie z.B. Kälteeinbrüche im Winter und Hitzewellen im Sommer.

Dünneres Eis verändert aber auch den Lebensraum in der Arktis. Weniger Transport-, Jagd- und Ruhemöglichkeiten bedrohen die Lebensweise der indigenen Bevölkerung und den Bestand an großen Säugetieren wie Walrossen, Robben und Eisbären. Da durch das dünne Eis mehr Sonnenlicht unter das Eis gelangt, wird die Masse der Kleinstlebewesen, wie Algen und Phytoplankton, die auf, im und unter dem Eis leben, wahrscheinlich zunächst wachsen. Langfristig könnte der Rückgang des Eises aber auch dazu führen, dass die Kleinstlebewesen weniger Nährstoffe zur Verfügung haben und sich dadurch ihre Produktion verringert. Phytoplankton braucht als Pflanze beim Wachsen $\mathrm{CO}_{2}$. Deshalb ist eine spannende, aber noch ungeklärte Frage, ob die Arktis in der Zukunft der Atmosphäre mehr $\mathrm{CO}_{2}$ entziehen oder an sie abgeben wird.

\section{Woher wissen wir, dass das Eis dünner wird?}

U-Boote und Satelliten messen wie tief das Eis ins Wasser eintaucht bzw. aus dem Wasser herausragt. Mit dem Prinzip von Archimedes und der Dichte von Meerwasser und Eis kann daraus die Gesamtdicke berechnet werden. Eine weitere Möglichkeit besteht darin, die Dicke per Flugzeug mit einem 


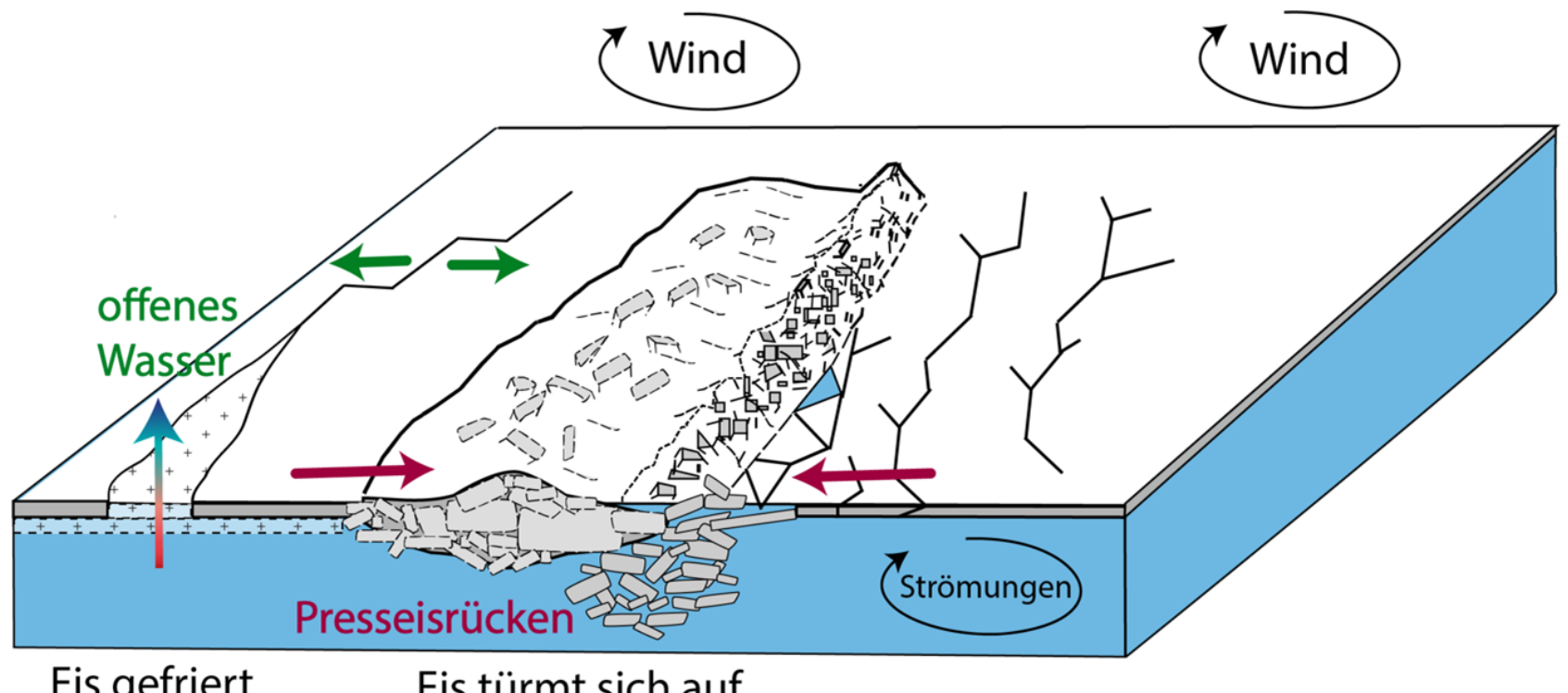

Eis gefriert Eis türmt sich auf

Abb. 1. Meereis wird dicker durch (1) Gefrieren an Stellen mit offenem Wasser und dünnem Eis und (2) Meereisdeformation. Wenn sich die Eisschollen aufeinander zubewegen, türmen sie sich auf und schieben sich übereinander (Abbildung: Luisa von Albedyll).

Instrument zu messen, das ähnlich wie ein Metalldetektor arbeitet. Es kann die Distanz zu dem Meerwasser unter dem Eis messen, das durch den Salzgehalt eine hohe elektrische Leitfähigkeit besitzt. Daraus berechnen Forschende zusammen mit dem Abstand des Messgeräts zum Eis die Dicke des Eises.

\section{Warum wird das Eis dünner?}

Das Schwinden der Eisdicke in der Arktis kann zwei Ursachen haben: (1) Es schmilzt mehr Eis als sich bildet. (2) Das Eis treibt schneller aus der Arktis heraus und hat deshalb weniger Zeit dicker zu werden.

\subsection{Wärmere Luft- und Meerestemperaturen}

Durch den Klimawandel erhöht sich die Temperatur der Luft und des Ozeans. Dadurch schmilzt das Eis im Sommer früher und gefriert später im Herbst. Auf lange Sicht wird die Arktis im Sommer eisfrei sein.

\subsection{Schnellere Eisbewegung}

Ein großer Teil des Eises bewegt sich angetrieben von Wind und Ozeanströmungen von seiner Kinderstube in den sibirischen Randmeeren über den Nordpol nach Grönland - die sogenannte Transpolardrift. Dort verlässt es die Arktis in Richtung Nordatlantik und schmilzt. Das Eis bewegt sich jetzt schneller auf dieser Transportroute. Hat es früher oft mehr als einen Winter in der Arktis erlebt, erreicht es den Nordatlantik jetzt schon häufig nach nur einem Jahr und hat dadurch weniger Zeit, dicker zu werden.

\section{Warum bewegt sich das Eis schneller?}

An dieser Frage forschen die Wissenschaftler gerade. Möglicherweise gelangen durch eine Veränderung in den großen Windsystemen häufiger starke Stürme bis zum Nordpol, die das Eis antreiben. Eine andere Erklärung liegt im dünnen Eis selbst. Das dünne Eis ist weniger belastbar und zerbricht schneller in kleinere Schollen, die der Wind leichter bewegen kann.

\section{Gibt es auch Prozesse, die eine Abnahme der Dicke abschwächen können?}

Ja, denn dünnes Eis wächst schneller als dickes Eis. Das liegt daran, dass dickes Eis und Schnee den Ozean von der kalten Luft isolieren und dadurch das Wachstum verlangsamen. Wenn das Eis in der Zukunft dünner ist, dann wächst im Winter schneller neues Meereis nach. Dieser Effekt bewirkt, dass im Winter die Meereisausdehnung nicht ganz so schnell abnimmt, wie in anderen Jahreszeiten. Das sorgt jedoch auch nicht für dickeres Eis.

Auch das häufigere Aufbrechen des dünnen Eises kann dazu führen, dass die Eisdicke lokal wieder zunimmt. Wenn das Eis bricht, schieben sich die Schollen aufeinander und türmen sich auf (Abb.1). Dabei entstehen große Eis-Barrikaden, die man Presseisrücken nennt und die so dick wie ein kleines Hochhaus werden können (Abb. 2). An einer anderen Stelle bricht das Eis auseinander und im Winter bildet sich in dieser Rinnen sofort dünne Schichten aus neuem Eis. Dadurch nimmt das Eisvolumen in der Arktis etwas zu. Wie groß der Einfluss von mehr Presseisrücken und Rinnen auf die Eisdicke in der Zukunft sein könnte, daran wird aktuell geforscht. 
(a)

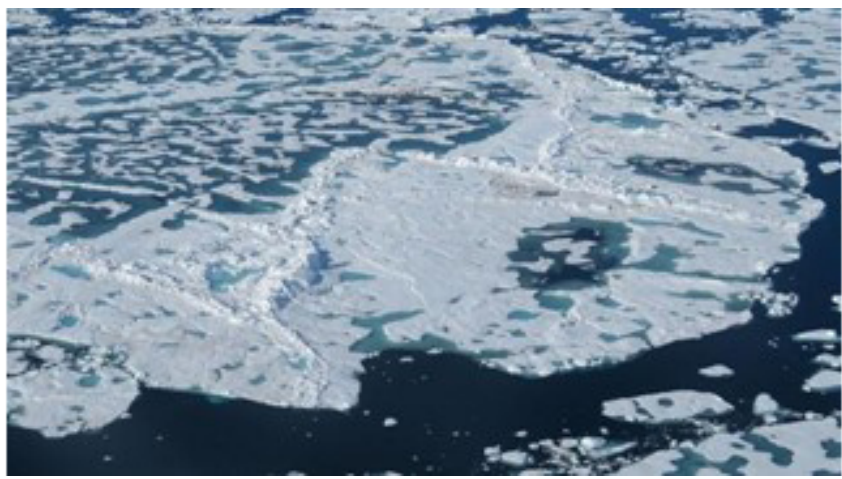

(b)

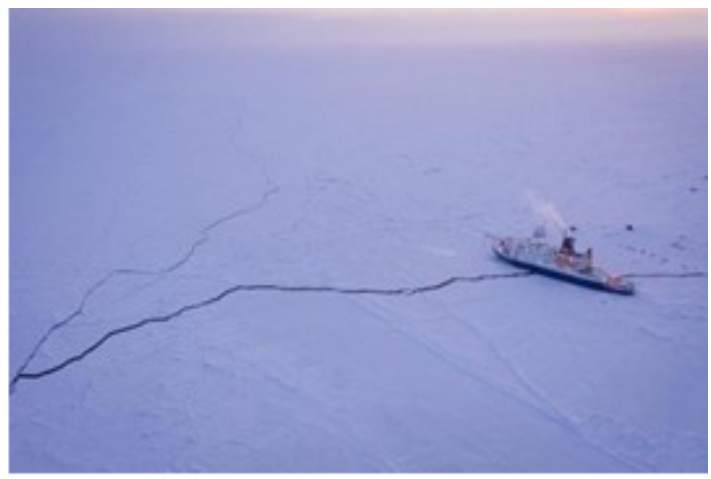

Abb. 2. (a) Ein Presseisrücken auf einer Eisscholle und (b) eine Rinne im Meereis in der Nähe des FS Polarstern (Foto: Luisa von Albedyll/Manuel Ernst/AWI).

\section{Wie geht es weiter?}

In den letzten 60 Jahren hat sich die mittlere Dicke des Meereises deutlich halbiert. Im Rahmen von großen Expeditionen wie MOSAiC in 2019/2020 und mit Hilfe von Satellitenbeobachtungen untersuchen Forschende die Effekte und Ursachen des dünner werdenden Meereises. Sie lassen ihre Ergebnisse in Computermodelle einfließen, die es uns ermöglichen, noch besser vorauszusagen wie die Zukunft des Meereises im Klimawandel aussehen wird.
Datenverfügbarkeit. Für diesen Artikel wurden keine Datensätze genutzt.

Interessenkonflikt. Die Autor*innen erklären, dass kein Interessenkonflikt besteht.

Begutachtung. This paper was edited by Donovan Dennis. 